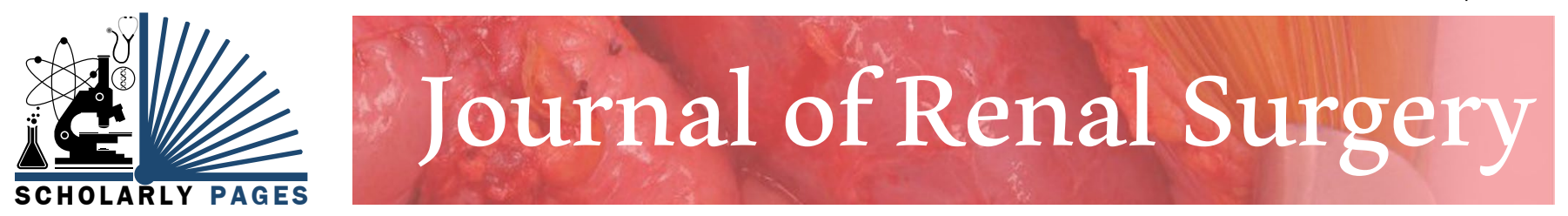

\title{
Nephron Sparing Surgery: A Run after a Chimera?
}

\section{Alexander Pycha ${ }^{1}$, Emanuela Trenti ${ }^{2}$, Salvatore Palermo ${ }^{2}$, EviComploj ${ }^{2}$, Christine Mian ${ }^{3}$, Esther Hanspeter ${ }^{3}$, Michael Mian ${ }^{4,5}$, Armin Pycha ${ }^{6 *}$ and EgilsVjaters ${ }^{1}$}

\author{
${ }^{1}$ Department of Urology, Riga Stradins University Hospital, Latvia \\ ${ }^{2}$ Department of Urology, Central Hospital of Bolzano, Italy \\ ${ }^{3}$ Department of Pathology, Central Hospital of Bolzano, Italy \\ ${ }^{4}$ Department of Hematology and Center of Bone Marrow Transplantation, Central Hospital of Bolzano, Italy \\ ${ }^{5}$ Department of Internal Medicine V (Haematology and Oncology), Medical University of Innsbruck, Austria \\ ${ }^{6}$ Chair of Urology, Sigmund Freud University Medical School, Austria
}

\begin{abstract}
Objective: To compare the long-term oncological and functional outcome as well as the surgical complications of nephron sparing surgery (NSS) versus radical nephrectomy (RN) for any renal cell carcinoma (RCC) over all stages (T1-T4).

Methods: From 2000 to 2016, 392 patients underwent renal surgery for RCC. 129 women and 263 men with a median age 65 (range 23-88) were operated. 162/392 (41.3\%) underwent a RN, 239/392 (58.7\%) a NSS. Clinical, surgical and pathological parameters were analyzed. Median follow-up time for these patients was 48.08 months (range 0.26-194.43).

Results: NSS showed a significantly higher disease free survival (DFS) $(70.2 \%$ vs. $93.5 \%, \mathrm{p}<0.001)$ and cancer specific survival (CSS) at 10 years $(78.4 \%$ vs. $97.8 \%, p=0.011)$, whereas the 10 years overall survival $(\mathrm{OS})$ in both groups did not differ significantly (RN $65.3 \%$ vs. NSS $71.3 \%, p=0.214$ ). Renal function preservation was better in the NSS group and the new onset of chronic kidney diseases was significantly less in the NSS group. Total complication rate was significantly less in the $\mathrm{RN}$ group (5.6\% vs. $8.9 \%$ ) but became comparable in the last years of observation.

Discussion: NSS was performed whenever technically possible but was obtained with a higher but acceptable surgical complication rate. It could be shown that also for higher stages of RCC, NSS can be safely performed. Renal function preservation, CSS and DFS were better in the NSS group but surprisingly NSS did not lead to a better OS. This stands in contrast to most published studies of the last decades.
\end{abstract}

Keywords

Renal cell cancer carcinoma, Radical nephrectomy, Nephron sparing surgery, Oncological outcome, Functional outcome, Co-morbidity

\section{Introduction}

Radical nephrectomy (RN) used to be the technique of choice for localized renal cell carcinoma (RCC) [1] with excellent oncological outcomes. The nephron sparing surgery (NSS) was limited to "so called imperative indications" as in case of bilateral RCCs as well as in tumours of a solitary kidney. With abdominal imaging more than $50 \%$ of RCCs are nowadays diagnosed incidentally $[2,3]$.

Surgical trends, based on a substantial number of retrospective studies [4-10] which indicated comparable oncological outcome but less de novo incidence of renal failure for NSS, moved towards renal preservation assuming that, preserving renal parenchyma, the non-cancer related mortality provoked by cardiovascular events could be prevented [11]. This survival benefit could be also the consequence of a selection bias as mentioned by Tomaszewski, et al. [12] because it seems that patients chosen for NSS possess a baseline higher likelihood of long term survival [13].

*Corresponding author: Armin Pycha, Department of Urology, General Hospital of Bolzano, Bolzano, Italy, Tel: +390471-908686,E-mail: armin.pycha@libero.it

Received: January 30, 2017; Accepted: April 08, 2017; Published online: April 10, 2017

Citation: Pycha A, Trenti E, Palermo S, et al. (2017) Nephron Sparing Surgery: A Run after a Chimera?. J Ren Surg 1(1):1-10

Copyright: ( 2017 Pycha A, et al. This is an open-access article distributed under the terms of the Creative Commons Attribution License, which permits unrestricted use, distribution, and reproduction in any medium, provided the original author and source are credited. 
Currently it is recommended by the EAU guidelines on RCC, that NSS should be performed for Tla and T1b tumours [14]. The indication for NSS, however, was extended also to larger tumours counting on long term benefits with comparable oncological outcome. To date 8 studies in T2 or greater are published and the results are controversal. In stages following the premise to perform NSS whenever technically possible, irrespectively of tumour size.

\section{Material and Methods}

This single-institution study ran from April 2000 to May 2016. Demographic and clinical-pathological characteristics of 392 patients, 129 women and 263 men, median age 65 (range 23-88) years, were collected prospectively and introduced in the institutional kidney cancer database. With the approval of institutional review board the data were retrospectively assessed.

Preoperative evaluation of all patients comprised laboratory including glomerular filtration rate (GFR) and cross sectional imaging of the chest and the abdomen to define the clinical tumour stage. A metastatic evaluation was also performed according to the current EAU Guidelines [14]. NSS was offered to all patients irrespectively of the tumour stage whenever it seemed technically feasible otherwise a RN was taken into consideration. All complications within 30 days of surgery were considered perioperative complications, all others as late complications. To classify the complications the Clavien Dindo classification (CDC) was used since 2005 [15].

Chronic kidney disease (CKD) stage was assigned according to the National Kidney Foundation definition [16]. Pathological data were also prospectively collected and included histological subtype, grade diameter and margin status. Tumours were staged according to the WHO classification of the International Society of Urological Pathology (ISUP) [17] and graded according to Fuhrmann until 2015 and according to WHO/ISUP starting in 2016 [17]. Tumour recurrence was defined as a new renal mass in the initial tumor bed in the absence of distant metastases based on CT-imaging.

Follow-up was calculated from the time of surgery until the last follow up (FU) visit or death. Mortality status was obtained through the Provincial Tumour Registry or by death certification of patients outside the province. Postoperative FU of patients included physical examination, laboratory examinations, chest $\mathrm{x}$-ray in two plains and an abdominal sonography every 3 months during the first two years, every 6 months from the second to the fifth year of follow-up and thereafter every year. The first CT control was performed three months after RN/ NSS and used as reference imaging. Thereafter every 6 months a CT scan of the kidneys was performed until the fifth year of follow up, then annually.

If any suspicion of disease progression arose, a complete restaging was performed.

\section{Surgical approach and technique}

Performed procedures included laparoscopy and open RN and NSS. Surgical modality and approach were decided by tumour characteristics, anatomical singularities and surgeons' preference. All surgeries were performed from or in the presence of two skilled surgeons (AP, SP).

\section{Endpoints and Statistical Analysis}

This observational study was designed to assess cancer control, functional outcome and surgical related complications. The primary outcomes were death or time to progression, with progression defined as local recurrence in either kidney or regional or distant metastases.

The analysis was performed using SPSS ver. 17.0 (SPSS Inc., Chicago, IL). Survival curves were plotted according to the Kaplan-Meier method. Overall survival (OS) was defined as time from diagnosis until death of any cause, cancer specific survival (CSS) as time from diagnosis until cancer related death and disease-free survival time (DSF) from the achievement of a complete remission until first recurrence [18]. Differences of categorical variables on survival were evaluated using the log-rank test. A multivariate analysis by cox regression was used to calculate the prognostic factor of tumour type, grade, stage, lymph node status, vein involvement, metastases, margins, diabetes and hypertension. A p-value of $<0.05$ was considered statistically significant. In univariate analysis, whenever possible, Fisher's Exact test was applied, otherwise the Pearson's Chi Square test.

\section{Results}

392 patients with 408 tumours, 129 women and 263 men, median age 65 (range 23-88) years, were included in the analysis. 162/392 (41.3\%) underwent RN, 230/392 NSS (58.7\%). 100 out of 392 patients (25.5\%) were operated laparoscopically, the other $292(74.5 \%)$ by open surgery. 227 tumours were located on the right kidney, 241 on the left one.

Median follow-up time for these patients was 48.08 (range 5.2-194.4) months. Baseline patient's characteristics showed no significant statistical differences between $\mathrm{RN}$ and NSS, although, a greater percentage of the RN group had American Society of Anesthesiologist (ASA) 3 or higher compared to the NSS group. Surgical parameters were similar between the groups even though a higher proportion of NSS was performed as open surgery. Patient's demographics and surgical data of both groups are given in (Table 1). 
Citation: Pycha A, Trenti E, Palermo S, et al. (2017) Nephron Sparing Surgery: A Run after a Chimera?. J Ren Surg 1(1):1-10

Table 1: Nephron sparing surgery versus radical nephrectomy: clinical, surgical and pathological parameters.

\begin{tabular}{|c|c|c|c|}
\hline Parameter & RN & NSS & p-value \\
\hline No of patients/No of tumours & 162 & $230 / 246$ & \\
\hline Women/men & $62 / 100$ & $67 / 169$ & 0.039 \\
\hline Median age (range) & $65(26-88)$ & $67(23-87)$ & 0.094 \\
\hline BMI & $26.4(18-44)$ & $24.8(19.5-37)$ & 0.68 \\
\hline ASA $3+4$ & $68(41.9 \%)$ & $78(33.9 \%)$ & 0.77 \\
\hline Patients with diabetes & $25(15.4 \%)$ & $31(13.5 \%)$ & 0.56 \\
\hline Patients with hypertension & $81(50 \%)$ & $123(53.5 \%)$ & 0.48 \\
\hline Preop. creatinine (mg/dl) & $1.0(0.6-3.4)$ & $0.94(0.56-3.5)$ & 0.811 \\
\hline Postop. creatinine (mg/dl) & $1.3(0.4-4.3)$ & $1.12(0.56-4.58)$ & 0.004 \\
\hline Last FU creatinine & $1.2(0.6-8.8)$ & $1.08(0.57-8.51)$ & 0.002 \\
\hline Preop. GFR (ml/min/1.73 m²) & $62(13-146)$ & $67.5(13-109)$ & 0.115 \\
\hline Postop. GFR (ml/min/1.73 m²) & $51(13-234)$ & $59.5(11-113)$ & $<0.001$ \\
\hline Last FU GFR (ml/min/1.73 $\left.\mathrm{m}^{2}\right)$ & $54(1.73-144)$ & $65(6-113)$ & $<0.001$ \\
\hline CKD stage I/II/III/IV/V & $11 / 90 / 55 / 5 / 1$ & $25 / 140 / 61 / 3 / 1$ & 0.648 \\
\hline Pathological tumour size; median (range) & $6.2(0.3-20)$ & $3(0.2-18)$ & $<0.001$ \\
\hline \multicolumn{4}{|l|}{ Pathology } \\
\hline Clear-cell & $136(83.9 \%)$ & $156(63.4 \%)$ & \multirow[t]{4}{*}{$<0.001$} \\
\hline Papillary & $13(8.0 \%)$ & $73(29.7 \%)$ & \\
\hline Chromophobe & $6(3.7 \%)$ & $12(4.9 \%)$ & \\
\hline Others & $7(4.3 \%)$ & $5(2.0 \%)$ & \\
\hline \multicolumn{4}{|l|}{ Tumor staging } \\
\hline pT1 & $79(48.7 \%)$ & $223(90.6 \%)$ & \multirow[t]{10}{*}{$<0.001$} \\
\hline pT1a & $27(16.6 \%)$ & $183(74.3 \%)$ & \\
\hline pT1b & $52(32.1 \%)$ & $40(16.3 \%)$ & \\
\hline pT2 & $28(17.2 \%)$ & $11(4.4 \%)$ & \\
\hline pT2a & $23(14.1 \%)$ & $6(2.4 \%)$ & \\
\hline pT2b & $5(3.1 \%)$ & $5(2.0 \%)$ & \\
\hline pT3 & $50(30.8 \%)$ & $11(4.4 \%)$ & \\
\hline pT3a & $33(20.3 \%)$ & $11(4.4 \%)$ & \\
\hline pT3b & $17(10.5 \%)$ & - & \\
\hline pT4 & $3(1.8 \%)$ & $1(0.4 \%)$ & \\
\hline N1 (5\%) & $4(2.4 \%)$ & $1(0.4 \%)$ & \multirow[t]{2}{*}{0.020} \\
\hline N2 & $1(0.6 \%)$ & - & \\
\hline M1 & $16(9.8 \%)$ & $7(2.8 \%)$ & 0.002 \\
\hline
\end{tabular}

Table 2: Oncological outcome.

\begin{tabular}{|l|l|l|l|}
\hline Parameter & RN & NSS & p-value \\
\hline Follow-up, median (range); years & $6.9(0.07-16.25)$ & $8.38(0.02-15.5)$ & $<0.001$ \\
\hline Positive margins (\%) & $2(1.2 \%)$ & $10(4.1 \%)$ & 0.210 \\
\hline Estimated DFS & & & \\
\hline 5-year (\%) & 81.8 & 98.0 & 0.011 \\
\hline 10-year (\%) & 70.2 & 93.5 & $<0.001$ \\
\hline Time to recurrence, median & 6.79 & 3.81 & 0.011 \\
\hline Estimated CSS & & & \\
\hline 5-year (\%) & 85.2 & 98.5 & \\
\hline 10-year (\%) & 78.4 & 97.8 & \\
\hline Estimated OS & & & \\
\hline 5-year (\%) & 80.0 & 96.7 & \\
\hline 10-year (\%) & 65.3 & 71.3 & \\
\hline
\end{tabular}

\section{Tumour characteristics, progression and survival}

Median diameter of the tumours was $3.5 \mathrm{~cm}$ (range $0.2-20 \mathrm{~cm}$ ). Histological subtype and tumour grade were similar between the groups (Table 1). The NSS group showed a greater proportion of T1 tumours (223 vs. 79 ) whereas the RN group had a higher proportion of $\mathrm{T} 3 / 4$ tumours ( 53 vs. 12 ). In $1.5 \%$ the lymphnodes were positive affecting only the RN group. $5.6 \%$ of the cases had metastases at the time of surgery, 16 in the RN group and 7 in the NSS group. Oncological outcomes are displayed in (Table 2). 

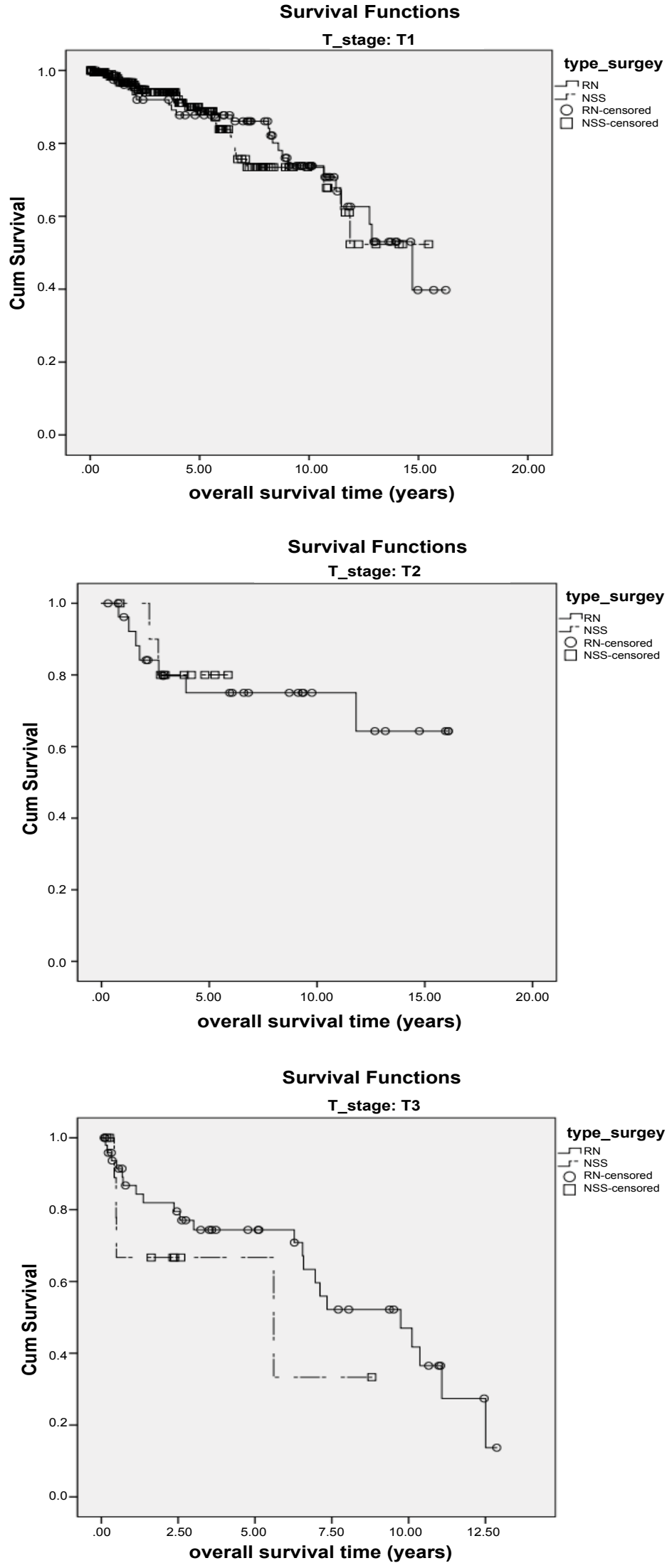

Figure 1: Kaplan-Meier analysis of overall survival time according to tumor stages. Median OS was 14.7 years after RN vs. not reached after NSS for T1, not reached in both for T2, 9.7 vs. 5.6 for T3 and not reached vs. 0.9 for T4, respectively. The different surgical approaches did not significantly influence overall survival (OS) according to the different disease stages at time of diagnosis. 
In total 88 out of 392 patients $(22.4 \%)$ died after a median of 90 months (2-180). Of the 162 patients who underwent RN, 58 (35.8\%) died after a median 91.5 months (range 2-171), 31 (19.1\%) cancer related. Of the $230 \mathrm{pa}$ tients who underwent NSS, 30 (13\%) died after a median 68.5 months (range 5-180), 8 (3.4\%) from cancer.

OS in the RN group was $80.0 \%$ after 5 years and $65.3 \%$ after 10 years, while it was $96.7 \%$ and $71.3 \%$, respective$l y$, in the NSS group $(p=0.214)$ (Table 2). If OS is stratified according to tumor stages the median OS in T1 was 14.7 years for RN and not reached for NSS, not reached in both for T2, whereas for T3 tumors the median OS in RN was 9.7 years versus 5.6 in NSS. Due to the low number of patients with T4 disease, it was not possible to draw any conclusion (Figure 1).

CSS in the RN group was $85.2 \%$ after 5 years and $78.4 \%$ after 10 years, in the group of patients with NSS it was $98.5 \%$ after 5 years and $97.8 \%$ after 10 years $(\mathrm{p}<0.001)$ (Table 2). If CSS is stratified according to tumor stages the median CSS was not reached for T1 and T2 independently of the type of surgery whereas in T3 tumors the median CSS was 9.7 years for RN vs. 5.6 years for NSS. Again in T4 tumors the number was too low to give a reliable evaluation (Figure 2).

In the RN group, $81.8 \%$ of the patients were disease free after 5 years and $70.2 \%$ after 10 years, in the NSS group, it was $98.0 \%$ and $93.5 \%$, respectively $(\mathrm{p}=0.011)$ (Table 2).

Of the 246 enucleated tumours 227 (92.3\%) had a negative resection margin, $10(4 \%)$ had a positive. $4(1.8 \%)$ tumours with negative margins showed recurrence after a median 50 months (6-94). 16 (7\%) of these tumours developed metastases after a median 16.5 months (2-79). Of 10 tumours with a positive resection margin 1 developed recurrence within 23 months.

Of the 162 patients who underwent RN, 153 (94.4\%) had a negative resection margin, 2 had a positive. 3 out of the 153 patients with negative margin showed recurrence after a median 123 months (range 122-177). 43 (28.6\%) of these patients developed metastases after a mean 40 months (1-126). Two patients with a positive resection margin developed metastases within 3 and 4 months.

\section{Functional outcome}

In both groups, creatinine levels (1.0 vs. 0.94$)$ and filtration rate (GFR) (62 vs. 67.5) were similar before surgery, while immediately after surgery and at last follow up NSS showed a significantly better functional preservation.

Details are given in (Table 1).
Table 3: Perioperative complications.

\begin{tabular}{|l|l|l|}
\hline Type of complications, $\mathbf{n}=\mathbf{3 2}$ & $\mathbf{R N} \mathbf{8} \mathbf{( 5 . 0 \% )}$ & $\begin{array}{l}\text { NSS 24 } \\
\mathbf{( 1 0 . 4 \% )}\end{array}$ \\
\hline Hematoma & $4(2.5 \%)$ & $1(0.4 \%)$ \\
\hline Urinoma & - & $7(3.0 \%)$ \\
\hline AV-fistula & - & $5(2.1 \%)$ \\
\hline Others & $4(2.5 \%)$ & $11(4.7 \%)$ \\
\hline Clavien Dindo grade I + II & $5(3.0 \%)$ & $4(1.7 \%)$ \\
\hline Clavien Dindo grade III a + b & $2(1.25 \%)$ & $18(7.8 \%)$ \\
\hline Clavien Dindo IV a + b & $1(0.6 \%)$ & $2(0.8 \%)$ \\
\hline
\end{tabular}

56 out of 392 patients (14.3\%) had diabetes, in the $\mathrm{RN}$ group 25 of $162(15.4 \%)$, in the NSS group, 31 of 230 (13.5\%) patients.

204 out of $392(52.0 \%)$ patients suffered of hypertension, in the RN group, 81 of 162 patients $(50 \%)$, in the NSS group 123 of 230 (53.5\%).

A total 42 out of 392 patients (10.7\%) suffered from both, diabetes and hypertension, 19/162 (11.7\%) in the $\mathrm{RN}$ group and 23/230 (10\%) in the NSS group.

\section{Complications}

In total, 33/392 patients (8.4\%) had complications after surgery, $10 / 162(6.2 \%)$ of the RN group and 23/230 $(10 \%)$ of the NSS group. The complications were grouped as hematomas, urinomas, arterio-venous (AV) fistulas and others (i.e. acute renal insufficiency, effusions, pneumonia) (Table 3).

\section{Discussion}

To our knowledge, this is the largest case control study to date comparing RN and NSS with the premise to perform NSS whenever technically possible, irrespectively of the tumour size ranging from $\mathrm{T} 1$ to $\mathrm{T} 4$ stages. It led to NSS being performed for $60.3 \%$ of all RCC which is a relatively high percentage compared with the US where still $77.6 \%$ of all T1a tumours are treated with RN [17]. Also in Europe, as shown by Aben, et al. [18], 70\% of T1b RCC undergo RN. Obviously there are some caveats in considering the EAU and AUA Guidelines.

Our results showed highly significant better CSS and DFS for NSS at five years and at ten years but not for OS after 5 years $(96.7 \%$ vs. $80 \%)$ and at ten years $(71.3 \%$ vs. $65.3 \%)$. The different surgical approaches did not significantly influence OS according to the different disease stages at time of diagnosis. While the survival curves were nearly overlapping for stage $\mathrm{T} 1$ and $\mathrm{T} 2$, in patients with T3, the OS after NSS was slightly inferior without achieving statistical significance. For T4 tumors the number was too small to draw a reliable conclusion. The statistical tendency, however, is in favour of NSS (Figure 3). The comparison of these results with published data surely indicates some imbalances. First, because our series has 
Citation: Pycha A, Trenti E, Palermo S, et al. (2017) Nephron Sparing Surgery: A Run after a Chimera?. J Ren Surg 1(1):1-10
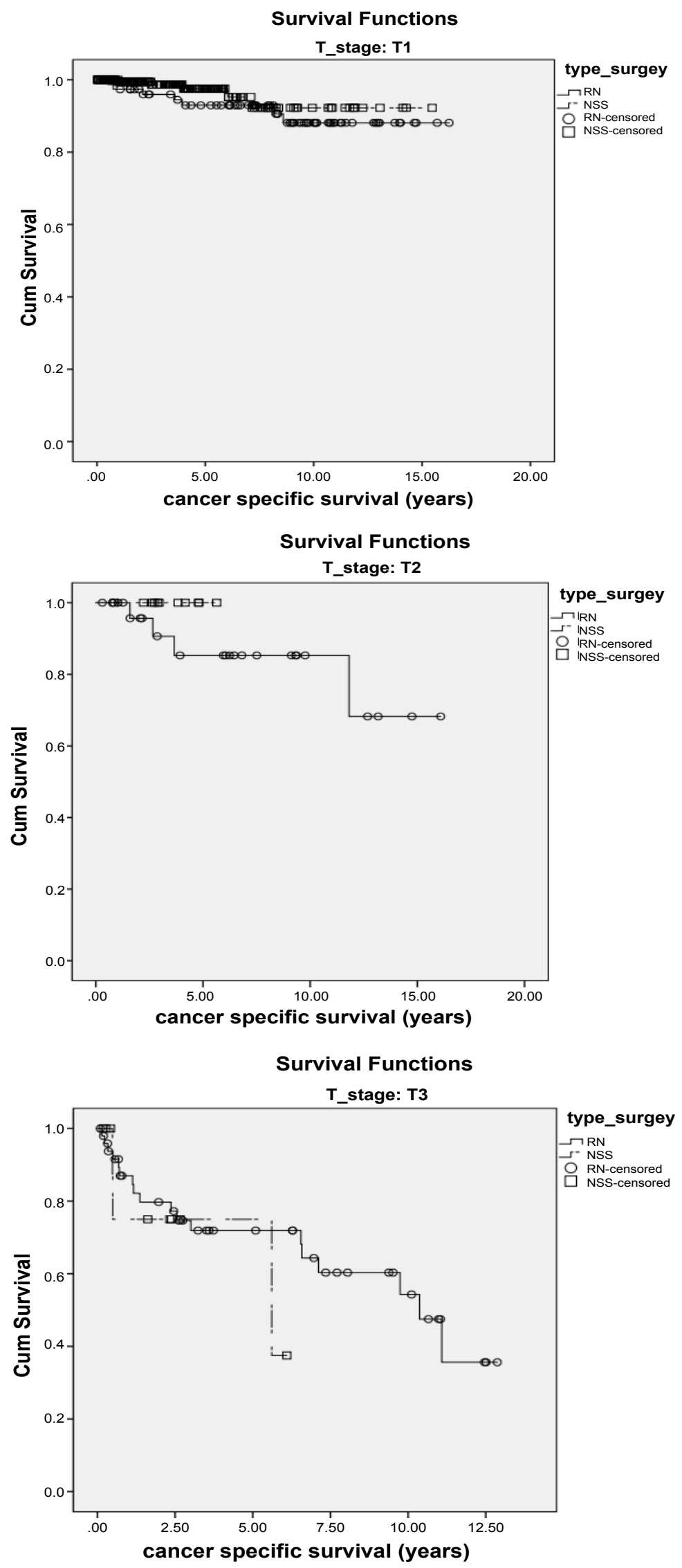

Figure 2: Kaplan-Meier analysis of cancer specific survival time (CSS) according to tumor stages. Median CSS was not reached for T1 and T2 independently of the type of surgery, 10.3 years after RN vs. 5.6 after NSS for T2, 9.7 vs. 5.6 after T3 and not reached vs. 2.08 for T4, respectively. As in OS, the different surgical approaches did not significantly influence CSS according to the different disease stages at time of diagnosis. 
no restriction regarding tumour size and comprises all $\mathrm{T}$-stages and second because the evidence in literature is limited to 34 studies fulfilling the PRISMA criteria. All these studies are differing in tumour size and FU available and are contradictory. D'Armiento, et al. [10] found an equal median survival at 98 months FU, [19] reported a statistically not significant survival benefit for $\mathrm{RN}$ at 5 years ( $80 \%$ vs. $75 \%)$ whereas Lee, et al. [20] demonstrated a tendency in favour of NSS at five years. The only prospective and randomized study from Van Poppel [21] showed inferior 10 years OS for NSS.

Tumour progression was found in $8.9 \%$ in patients of the NSS group. Of those, 6 (2.4\%) patients developed a recurrence in the previous operated kidney and 16 patients $(6.5 \%)$ distant metastases after a median duration of 16.5 months. In the literature the local recurrence rate ranges between $4 \%-6 \%$ with few exceptions as in the study from Herr who reports $1.4 \%$ local recurrence rate at 10 years [8] or in the Swedish population based study where local recurrences reach $20 \%$ [22]. In the Bolzano study the rate of distant metastases was $6.5 \%$, which is in the middle of the in the literature reported range of $5.8 \%$ and $8.6 \%$ [1] for NSS. Once again, all the studies are biased by different tumour stages, tumour sizes, and different follow-ups.

In the RN group $28.4 \%$ of the patients experienced a progression. Local recurrence was seen only in 3 (1.9\%) patients. It is similar to the Liebovich study [23] where the rate was $2.2 \%$. The $\mathrm{RN}$ group includes in both studies a higher percentage of advanced $\mathrm{T}$-stages and positive lymph nodes in the final pathology, so it could be argued that the local recurrence in NSS could be largely avoided with radical surgery.

Achieving a negative surgical margin (NSM) during NSS is a fundamental principle of oncological surgery and the best prevention of local recurrence, but does a positive surgical margin (PSM) necessarily lead to a recurrence and subsequently to a systemic progression and finally to a cancer related mortality? In the NSS group 10 (4\%) patients had a PSM in the final histo pathological report. One $(0.4 \%)$ of them developed a local recurrence but none of them a distant metastasis which is in accordance with the current literature where the range for PSM is between $4 \%$ and $10 \%$. PSM after NSS will not always result in a local recurrence and /or disease progression. It is suggested that a PSM should not be sanctioned immediately by a secondary nephrectomy. A very close follow-up is mandatory [24,25].

The situation is different when it comes to RN. 2 (1.2\%) patients had a PSM, none developed local recurrence but both suffered from metastases. It reflects the tumour biology. If RN cannot anymore be performed with NSM it reflects an advanced tumour stage (stage III or IV) mostly combined with a high biological aggressiveness of the tumour. Both are negative predictors regarding OS and CSS. In the recent study the metastases rate in NSM group of RN was seen in $43(28.1 \%)$ patients after a median duration of 19.5 months. It is nearly the same as in the Swedish population based study [22] where $19.9 \%$ out of 2858 patients with all tumour stages, treated with $\mathrm{RN}$, developed distant metastases after a mean time of 24 months [22]. Both studies have in common that patients with recurrence had significantly larger tumour size, more often T2-T4 tumours and had a higher number of Grade 3-4. Presence of distant metastases was significantly more common after RN than after NSS in all stages. Our findings concerning the progression after RN compared to NSS were probably a result of the selection of patients with smaller and less aggressive tumours for NSS.

Our series failed to evidence an oncological benefit for NSS in higher tumor stages (Figure 3).

We could demonstrate a superior renal function preservation and less new onset of CKDs for NSS in our cohort compared to many other studies $[1,3,26]$. The percentage decline of GFR from baseline to postoperative follow-up time was significantly smaller in patients treated with NSS (3.7\%) compared with RN (12.9\%). Both groups experienced a decline in renal function immediately after the operation, then a slight recovery of the GFR with a long lasting stable run, a phenomenon not observed in other studies but one [2]. Although the risk of moderate $\mathrm{CKD}$ in the elective setting is higher with $\mathrm{RN}$, the risk of end-stage renal disease (ESRD) is limited after elective RN or NSS. This finding is confirmed by the EORTC 30904 trial [21] where the risk is quantified with $0.8 \%$ NSS and $1.2 \%$ for RN. The same affirmation cannot be made for patients with preoperative CKD. There the risk of ESRD increases dramatically [12,27].

NSS is considered a riskier operation than $\mathrm{RN}$ due to the combination of an ablative surgical phase followed by a reconstructive part. More centrally situated tumours require the reconstruction of the collecting system, the control of potential bleeding sources and the adaption of the parenchyma to prevent bleeding and leakage. All these complications are avoidable with RN.

Several studies showed that when comparing the complication rate between NSS and RN there was a tendency towards a higher complication rate after NSS. $[1,2,7,8,21,22,28,29]$. In the literature the complication rate for open NSS for T1 RCC ranges between 4.5\%-8.7\% and increases to $18.8 \%$ for tumours $>7 \mathrm{~cm}$, not including the transfusion rate of up to $8.2 \%$ [1]. The best evidence was obtained from the prospective study of (EORTC 30904) [19] with an evidence level I-b. There NSS was associated with a higher risk of severe perioperative bleed- 

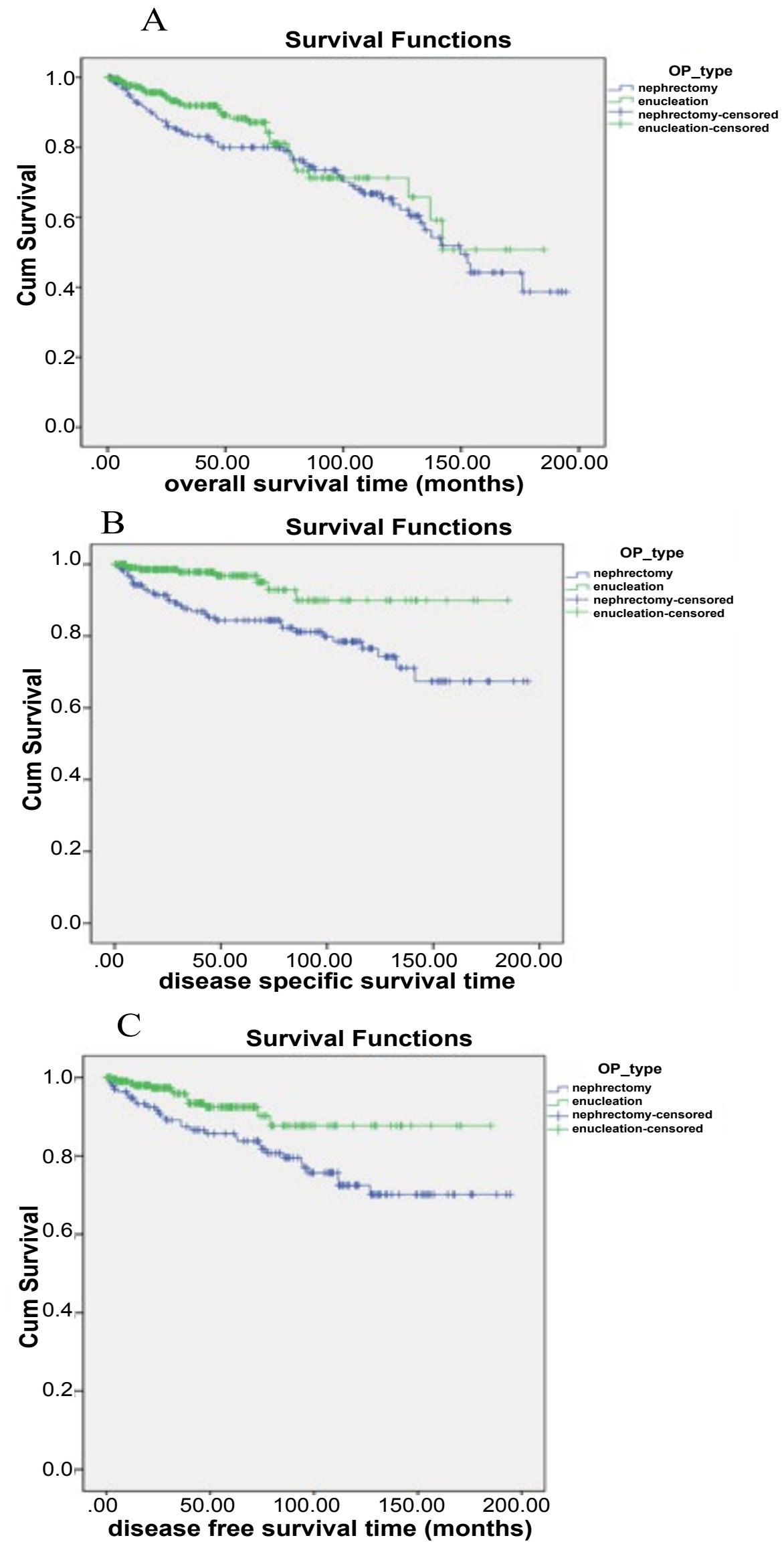

Figure 3: Kaplan-Meier analysis of overall survival time (A) cancer specific survival (B) and disease free survival (C) for 392 patients after radical nephrectomy or nephron sparing surgery for renal cell cancer of all stages. Comparison of survival analysis performed using the long-rank test. 
ing ( $3.1 \%$ vs. $1.2 \%)$, reoperation $(4.4 \%$ vs. $2.4 \%)$, urine fistula $(4.4 \%$ vs. $0 \%)$, pleura damage ( $11.5 \%$ vs. $9.3 \%)$. In the Bolzano cohort the total complication rate achieved $8.1 \% .5 .5 \%$ of the patients in the RN group experienced a complication, $10 \%$ in the NSS (blood transfusions not included) which is statistically significant $(\mathrm{p}<0.05)$. The peri operative bleeding rate was $0.8 \%$ in the NSS group vs. $2.5 \%$ in the $\mathrm{RN}$ group. Here the higher stages in the $\mathrm{RN}$ group are responsible for this selection bias. Due to the paucity of the group a matched pair analysis is not feasible. In all other complications the NSS group is burdened with up to a 3-fold higher complication rate, but the Bolzano experience is in line with the most published studies $[1,2,21]$ and shares a common observation. With increasing experience in NSS the complication rate decreased and approaches the one of the RN group.

Despite showing several strengths, as e.g. the large study cohort, the long observation period over 15 years, the always identical surgical strategy, the presence of one of two surgeons (AP and SP) in the theatre in all cases, guaranteeing the technical continuity and uniformity of the surgical procedure, the large median tumour size compared to other studies, our study implicates some limitations.

First and most importantly, it is limited by its observational nature and its retrospective design. Therefore, it is suitable to generate a hypothesis but not evidence at high level. Moreover, there may have been other factors impacting survival rates in both groups as selection bias and unobserved but measured confounders. Although the emerging data from the last decades about $\mathrm{RN}$ compared with NSS are contradictory, the present study is valuable because it adds the evidence of feasibility of NSS even for large and for all RCC stages, but better CSS and DFS for NSS lead not to longer OS.

\section{Conclusion}

This retrospective study, including 392 patients undergoing NSS whenever technically possible, could show that NSS can be safely performed for higher stages of RCC. The surgical complications are higher in the NSS group. Their frequency is acceptable and decreased over time, approaching the complication rate of the $\mathrm{RN}$ group over the last years. The risk of local recurrence, even in presence of PSM, is minimal.

Despite a better CSS and DFS in the NSS group, this study could not demonstrate an OS benefit in NSS, especially for higher tumour stages.

\section{References}

1. Long CJ, Canter DJ, Kutikov A, et al. (2012) Partial nephrectomy for renal masses $>7 \mathrm{~cm}$ : technical, oncological and funcional outcome. BJU international 109: 1450-1456.
2. Jang HA, Kim JW, Byun SS, et al. (2016) Oncological and functional outcomes after partial nephrectomy versus radical nephrectomy in T1b renal cell carcinoma: a multicenter, matched case-control study in korea patients. Cancer Res Treat 48: 612-620.

3. Forbes CM, Rendon RA, Finelli A, et al. (2016) Disease progression and kidney function after partial vs. Radical nephrectomy for T1 renal cancer. Urologic Oncology 34: 486. e17-486.e23.

4. Uzzo RG, Novick AC (2001) Nephron sparing surgery for renal tumours: indications techniques and outcomes. J Urol 166: 6-18.

5. Patard JJ, Shvarts O, Lam JS, et al. (2004) Safety and efficacy of partial nephrectomy for all T1 tumours based on an international multicenter experience. J Urol 171: 2181-2185.

6. Becker F, Siemerst S, Hack M, et al. (2006) Excellent longterm cancer control with elective nephron-sparing surgery for selected renal cell carcinomas measuring more than 4 cm. Eur Urol 49: 1058-1064.

7. Lau WK, Blute ML, Weaver Al, et al. (2000) Matched comparison of radical nephrectomy vs nephron sparing surgery in patients with unilateral renal cell carcinoma and a normal contralateral kidney. Mayo Clin Proc 75: 1236-1242.

8. Herr HW (1999) Partial nephrectomy for unilateral renal carcinoma and a normal contralateral kidney: 10-year follow-up. J Urol 161: 33-34.

9. James McKiernan, Rachel Simmons, Jared Katz, et al. (2002) Natural history of chronic renal insufficiency after partial and radical nephrectomy. Urology 59: 816-820.

10. D'Armiento M, Damiano R, Feleppa B, et al. (1997) Elective conservative surgery for renal carcinoma versus radical nephrectomy: a prospective study. $\mathrm{Br} \mathrm{J}$ Urol 79: 15-19.

11. Van Poppel H, Da Pozzo L, Albrecht W, et al. (2007) A prospective randomized EORTC intergroup phase 3 study comparing the complications of elective nephron-sparing surgery and radical nephrectomy for low-stage renal cell carcinoma. Eur Urol 51: 1606-1615.

12. Jeffrey J Tomaszewski, Alexander Kutikov (2015) Retrospective comparison of cardiovascular risk in preselcted patients undergoing kidney cancer surgery: reflection of reality or simply what we want to hear? Eur Urol 67: 690-691.

13. Shuch B, Hanley J, Lai J, et al. (2013) Overall survival advantage with partial nephrectomy: a bias of observationaldata? Cancer 119: 2981-2989.

14. Ljungberg B, Bensalah K, Canfield S, et al. (2015) EAU-Guidelines on renal cell carcinoma: 2014 update. Eur Urol 67: 913-924.

15. Dindo D, Demartines N, Clavien PA (2004) Classification of surgical complications: a new proposal with evaluation in a cohort of 6336 patients and results of a survey. Ann Surg 240: 205-213.

16. Levey AS, Eckardt KU, Tsukamoto Y, et al. (2005) Definition and classification of chronic kidney disease: a position statement from Kidney Disease: Improving Global Outcomes (KDIGO). Kidney Int 67: 2089-2100.

17. Moch H, Humphrey PA, Ulbright TM, et al. (2016) WHO classification of tumours of the urinary system and male genital organs. ( $4^{\text {th }}$ edn), Lyon : IARC Press.

18. EL Kaplan, Paul Meier (1958) Non parametric estimation from 
incomplete observations. J Am Stat Assoc 53: 457-481.

19. Butler BP, Novick AC, Miller DP, et al. (1995) Management of small unilateral renal cell carcinomas: radical versus nephron sparing surgery. Urology 45: 34-40.

20. Jin Hyung Lee, Chang Hee You, Gyeong Eun Min, et al (2007) Comparison of the surgical outcome and renal function between radical and nephron sparing surgery for renal cell carcinomas. Korean J Urol 48: 671-676.

21. Van Poppel H, DaPozzo L, Albrecht W, et al. (2011) A prospective, randomised EORTC intergroup phase 3 study comparing the oncological outcome of elective nephron sparing surgery and radical nephrectomy for low-stage renal cell carcinoma. Eur Urol 59: 543-552.

22. Dabestani S, Throstenson A, Lindblad P, et al. (2016) Renal cell carcinoma recurrences and metastases in primary non-metastatic patients: a population based study. WJU 34: 1081-1086.

23. Leibovitch I, Raviv G, Mor Y, et al. (1995) Reconsidering the necessity of ipsilateral adrenalectomy during radical nephrectomy for renal cell carcinoma. Urology 46: 316-320.

24. Hein Van Poppel, Steven Joniau (2007) How important are surgical margins in nephron sparin surgery? Eur Urol 6:
533-539.

25. Kwon EO, Carver BS, Snyder ME, et al. (2007) Impact of positive surgical margins in patients undergoing partial nephrectomy for renal cortical tumours. BJU Int 99: 286-289.

26. Kalagirou C, Fender H, Muck P, et al. (2017) Long term outcome of nephron sparing surgery compared to radical nephrectomy for renal cell carcinoma $\geq 4 \mathrm{~cm}$ - a matched-pair single institution analysis. Urol Int 98: 138-147.

27. Tobert CM, Riedinger CB, Lane BR (2014) Do we know (or just believe) that partial nephrectomy leads to better survival than radical nephrectomy for renal cancer? World J Urol 32: $573-579$.

28. Corman J, Penson DF, Hur K, et al. (2000) Comparison of complications after radical and partial nephrectomy: results from the National Veterans Administration Surgical Quality Improvment Program. Br J Urol 86: 782-789.

29. Shekarriz B, Upadhayay J, Shekarriz H, et al. (2002) Comparison of cost and complications of radical and partial nephrectomy for treatment of localized renal carcinoma. Urology 59: 211-215. 